JOURNAL OF

FUNCTION SPACES AND APPLICATIONS

Volume 6, Number 1 (2008), 59-70 (c) 2008, Scientific Horizon http://www.jfsa.net

\title{
Bounded holomorphic projections for exponentially decreasing weights
}

\author{
Wolfgang Lusky and Jari Taskinen* \\ (Communicated by Miroslav Englis)
}

2000 Mathematics Subject Classification. 46E15, 47B37.

Keywords and phrases. Projection, analytic function space, weighted norm, Cesaró summation.

Abstract. We construct generalized Bergman projections on a large class of weighted $L^{\infty}$-spaces. The examples include exponentially decreasing weights on the unit disc and complex plane.

\section{Introduction}

The classical Bergman projection $P: f \mapsto \int_{\mathbb{D}} f(\zeta)(1-z \bar{\zeta})^{-2} d A(\zeta)$, where $d A$ is the normalized 2-dimensional Lebesgue measure on the open unit disc $\mathbb{D}$ of the complex plane, is a bounded operator on $L^{p}(\mathbb{D})=L^{p}(\mathbb{D}, d A)$ for

* Research of Taskinen partially supported by the Academy of Finland Project 50957 and the Vaisala Foundation of the Finnish Academy of Sciences and Letters. 
$1<p<\infty$; it projects the space onto its closed subspace of analytic functions. In the case $p=1$, any projection

$$
P_{\alpha}: f \mapsto(\alpha+1) \int_{\mathbb{D}} \frac{f(\zeta)\left(1-|\zeta|^{2}\right)^{\alpha}}{(1-z \bar{\zeta})^{2+\alpha}} d A(\zeta), \quad \alpha>0
$$

has the same role, but for $p=\infty$, no analogous bounded projection exists. See [8] for these classical facts. However, with weighted sup-norms the situation is different. It is easy to see that for every $\beta>0$, the projection $P_{\alpha}$ is bounded for $\alpha+1>\beta>0$ on the space $L_{v}^{\infty}(\mathbb{D})$ of measurable functions bounded with respect to the weighted sup-norm

$$
\|f\|_{v}:=\operatorname{ess} \sup _{z \in \mathbb{D}} v(z)|f(z)|, \quad \text { where } v(z):=(1-|z|)^{\beta} .
$$

Other Bergman-type projections and also non-radial weights were recently considered in [2]. For more results, see [1], [5], [7], [8].

Not much is known about bounded projections in the case of weighted $L^{p}$-norms $\left(\int|f|^{p} v d A\right)^{1 / p}$, if the weight function decreases rapidly as a function of the boundary distance, e.g. $\left.v(z):=\exp \left(-1 / 1-|z|^{2}\right)\right), z \in \mathbb{D}$. In this case, the orthogonal projection of the space $L_{v}^{2}(\mathbb{D})$ is known to be unbounded in the spaces $L_{v}^{p}(\mathbb{D})$ for $p \neq 2$, and it seems that no bounded projection from $L_{v}^{p}(\mathbb{D})$ onto its subspace of analytic functions is known.

However, the first named author proved recently in [6] that for a large class of weights satisfying a condition $(B)$, see below, the space $H v$ (the subspace of $L_{v}^{\infty}(\mathbb{D})$ consisting of analytic functions) is isomorphic as a Banach space to $\ell^{\infty}$. This implies, by [4], p.105, the existence of a bounded projection from $L_{v}^{\infty}$ onto $H v$. The exponential weight mentioned above satisfies the condition $(B)$.

It is of course of interest to find out concrete formulas for such projections. This task is carried out in the present paper. We construct "canonical" bounded projections from $L_{v}^{\infty}$ onto the subspace $H v$ of analytic functions for weights satisfying the condition $(B)$. It is possible to use these projections to create a satisfactory theory of Toeplitz operators on these weighted spaces, see [3].

The projections will have series representations. To descibe them shortly in the case of $\mathbb{D}$, we shall associate to a given weight an increasing sequence $\left(s_{n}\right)_{n=1}^{\infty}, 0<s_{n}<1$, and a sequence $\left(T_{n}\right)_{n=1}^{\infty}$ of finite rank operators, which are just multipliers on the sequence space of Fourier coefficients. (They essentially arise from two Cesaró summations.) If $f: \mathbb{D} \rightarrow \mathbb{C}$ is a continuous function, we form for each radius $r \in] 0,1\left[\right.$ the Fourier-coefficients $f_{k}(r)$ of 
$\left.f\right|_{r \partial \mathbb{D}}$ and write

$$
f(z)=\sum_{k=-\infty}^{\infty} f_{k}(r) e_{k}(z)
$$

which makes sense at least in $L^{2}$. Here $e_{k}(z):=r^{|k|} e^{i k \varphi}$ for $z=r e^{i \varphi}$. The projection $P_{C}$ is then formally given by

$$
\begin{aligned}
P_{C} f(z) & =\sum_{n=1}^{\infty} T_{n} \sum_{k \geq 0} f_{k}\left(s_{n}\right) e_{k}(z) \\
& =\sum_{k \geq 0}\left(\sum_{n=1}^{\infty} t_{n k} f_{k}\left(s_{n}\right)\right) z^{k} .
\end{aligned}
$$

The convergence of this series will be proven below. For each fixed $n$, there are only finitely many nonzero numbers $t_{n k}$; they are the coefficients of the multiplier operators $T_{n}$ see (6). Also, given a fixed degree $k$, there exist at most 2 nonzero $t_{n k}$. Actually $0 \leq t_{n k} \leq 1$, and, for an increasing sequence $\left(m_{n}\right)_{n=1}^{\infty}$, for a fixed $n$, the value of $t_{n k}$ grows linearly from 0 at $k \cong m_{n-1}$ to 1 at $k \cong m_{n}$, and then decreases back to 0 at $k \cong m_{n+1}$. This interplay of the degree $m_{n}$ on one hand, and the radius $s_{n}$ on the other hand, is of essential importance for the proof of the boundedness of the projection $P_{C}$.

If $f$ is just an $L^{\infty}$-function, the point evaluations of the coefficients $f_{k}$ have to be replaced by integral means.

It is probably difficult to find integral kernels in terms of elementary functions for $P_{C}$. One of the reasons is that the definition of the numbers $s_{n}$ and $m_{n}$ is not completely trivial.

\section{Preliminaries, notations}

We consider function spaces defined on $\Omega$ which is either $\mathbb{D}$ or $\mathbb{C}$. By a weight we mean a continuous, radial function $v: \Omega \rightarrow] 0, \infty[$, which is also strictly decreasing as $r:=|z|$ increases. We assume that $\lim _{r \rightarrow 1} v(r)=0$, if $\Omega=\mathbb{D}$, and $\lim _{r \rightarrow \infty} r^{m} v(r)=0$ for any $m \geq 0$, if $\Omega=\mathbb{C}$. We define the spaces

$$
\begin{aligned}
L_{v}^{\infty} & :=\left\{f: \Omega \rightarrow \mathbb{C} \text { measurable }\left|\|f\|_{v}:=\operatorname{ess} \sup _{z \in \Omega} v(z)\right| f(z) \mid<\infty\right\} \\
h v & :=h_{v}^{\infty}:=\left\{f: \Omega \rightarrow \mathbb{C} \text { harmonic } \mid\|f\|_{v}<\infty\right\} \\
H v & :=H_{v}^{\infty}:=\{f \in h v \mid f \text { holomorphic }\}
\end{aligned}
$$


We also denote, for functions $f$ on $\mathbb{D}$ or $\mathbb{C}$,

$$
M_{\infty}(f, r):=\sup _{|z|=r}|f(z)|
$$

We shall need some notations and results from [6]. In [6], the first named author showed the boundedness of the Riesz projection $R: \sum_{k=-\infty}^{\infty} f_{k} e_{k} \mapsto$ $\sum_{k \geq 0} f_{k} z^{k}$ from $h v$ onto $H v$ provided the following condition $(B)$ holds for the weight $v$ :

Definition 1. The weight $v$ satisfies the condition $(B)$, if

$$
\begin{aligned}
& \forall b_{1}>1 \exists b_{2}>1 \exists c>0 \forall m, n>0 \\
& \left(\frac{r_{m}}{r_{n}}\right)^{m} \frac{v\left(r_{m}\right)}{v\left(r_{n}\right)} \leq b_{1} \text { and } m, n,|m-n| \geq c \Rightarrow\left(\frac{r_{n}}{r_{m}}\right)^{n} \frac{v\left(r_{n}\right)}{v\left(r_{m}\right)} \leq b_{2}
\end{aligned}
$$

Note that $m$ and $n$ need not be integers. Here the number $r_{n}>0$ denotes the global maximum point of the function $r \mapsto r^{n} v(r)$. It is easy to see that $r_{n}<r_{m}$ for $n<m$ and that $r_{n}$ tends to the radius of the domain, as $n \rightarrow \infty$.

From now on we fix a weight $v$ having the property $(B)$.

We soon define (in Theorem 1 below) an increasing sequence $\left(m_{n}\right)_{n=1}^{\infty}$ of real numbers. Given such a sequence, we shall define the operators $T_{n}$ acting on harmonic functions $f(z):=\sum_{k \in \mathbb{Z}} f_{k} e_{k}(z)$ by

$$
\begin{aligned}
T_{n} f:= & \sum_{m_{n-1}<|k| \leq m_{n}} \frac{|k|-\left[m_{n-1}\right]}{\left[m_{n}\right]-\left[m_{n-1}\right]} f_{k} e_{k} \\
& +\sum_{m_{n}<|k| \leq m_{n+1}} \frac{\left[m_{n+1}\right]-|k|}{\left[m_{n+1}\right]-\left[m_{n}\right]} f_{k} e_{k} \\
= & : \sum_{k \in \mathbb{Z}} t_{n k} f_{k} e_{k}
\end{aligned}
$$

Here $[a]$ denotes the largest integer $\leq a$. So the operator $T_{n}$ is a multiplier on the space of Fourier-coefficients. Given a sequence $\left(m_{n}\right)_{n=1}^{\infty}$, we also denote

$$
s_{n}:=r_{m_{n}} .
$$

We collect in the following theorem everything that is needed from [6]. Recall that $v$ is assumed to satisfy $(B)$. 
Theorem 1. There are numbers $0<m_{1}<m_{2}<\ldots$ and constants $d_{1}, d_{2}>0$ such that for any $f \in h v$ we have

$$
d_{1} \sup _{n} M_{\infty}\left(T_{n} f, s_{n}\right) v\left(s_{n}\right) \leq\|f\|_{v} \leq d_{2} \sup _{n} M_{\infty}\left(T_{n} f, s_{n}\right) v\left(s_{n}\right)
$$

and

(9) $d_{1} M_{\infty}\left(T_{n} f, s_{n}\right) v\left(s_{n}\right) \leq\left\|T_{n} f\right\|_{v} \leq d_{2}\left(\sup _{k}\left\|T_{k}\right\|\right) M_{\infty}\left(T_{n} f, s_{n}\right) v\left(s_{n}\right)$

for all $n$.

The operators $T_{n}$ are uniformly bounded with respect to $M_{\infty}(\cdot, 1)$.

Finally, the Riesz projection $R: h v \rightarrow H v$ is bounded with respect to $\|\cdot\|_{v}$.

Remark 1. (i) Our operators $T_{n}$ are equal to $V_{m_{n+1}, m_{n}}-V_{m_{n}, m_{n-1}}$ of [6], see (3.1) in the reference. They have the properties $f=\sum_{n} T_{n} f$ for every trigonometric polynomial $f$, and

$$
T_{n} T_{m}=0
$$

for $|n-m| \geq 2$.

(ii) For any series $\sum_{k=0}^{\infty} f_{k} e_{k}, f_{k} \in \mathbb{C}$, for any $n$, only finitely many summands of $\sum_{k=0}^{\infty} T_{n} f_{k} e_{k}$ are nonzero. (Hence there are absolutely no convergence problems with the latter series.)

(iii) Theorem 1 implies that $\sum_{k=0}^{\infty} f_{k} e_{k}$ is the Taylor series (converging at least uniformly on compacta of $\mathbb{D}$ ) of an analytic function $f \in H v$, if the coefficients $f_{k}$ are such that

$$
\begin{aligned}
\text { (11) } \sup _{n} M_{\infty}\left(\sum_{k=0}^{\infty} T_{n} f_{k} e_{k}, s_{n}\right) v\left(s_{n}\right) & =\sup _{n} M_{\infty}\left(\sum_{k=0}^{\infty} t_{n k} f_{k} e_{k}, s_{n}\right) v\left(s_{n}\right) \\
& <\infty
\end{aligned}
$$

Proof of Theorem 1. We obtain the numbers $m_{n}$ from Lemma 5.1 of [6]. In particular, one of the quotients

$$
\left(\frac{s_{n}}{s_{n+1}}\right)^{m_{n}} \frac{v\left(s_{n}\right)}{v\left(s_{n+1}\right)} \quad \text { or } \quad\left(\frac{s_{n+1}}{s_{n}}\right)^{m_{n+1}} \frac{v\left(s_{n+1}\right)}{v\left(s_{n}\right)}
$$

is equal to the constant $b>0$ of the reference. Condition $(B)$ yields a constant $d>b$ (independent of $n$ ) such that both quotients are numbers in the interval $[b, d]$. Now, Proposition 5.2. of [6] yields constants $c_{1}, c_{2}>0$ 
such that

$$
\begin{aligned}
& c_{1} \sup _{n} \sup _{s_{n-1} \leq r \leq s_{n+1}} M_{\infty}\left(T_{n} f, r\right) v(r) \\
& \quad \leq\|f\|_{v} \leq c_{2} \sup _{n} \sup _{s_{n-1} \leq r \leq s_{n+1}} M_{\infty}\left(T_{n} f, r\right) v(r) .
\end{aligned}
$$

This already implies the first inequality of (8). Using [6], Proposition 4.1, we see that either $m_{n+1}-m_{n-1} \leq c$ for all $n$ ( $c$ as in condition $(B)$ ) or there are $\eta, \kappa>0$, independent of $n$, such that

$$
\eta \leq \frac{m_{n+1}-m_{n}}{m_{n}-m_{n-1}} \leq \kappa .
$$

Lemma 3.3.(c) of [6] then tells us that, in any case, the operators $T_{n}$ are uniformly bounded with respect to $M_{\infty}(\cdot, 1)$. (If $m_{n+1}-m_{n-1} \leq c$, then rank $T_{n} \leq c$.)

Using Lemma 3.1. of [6] we see that here

$$
\sup _{s_{n-1} \leq r \leq s_{n+1}} M_{\infty}\left(T_{n} f, r\right) v(r) \leq 2 b d M_{\infty}\left(T_{n} f, s_{n}\right) v\left(s_{n}\right) .
$$

Hence, the second inequality of (8) follows.

The first inequality in (9) is a triviality. Concerning the second, we use (10) and (12) to obtain for a fixed $n$

$$
\begin{aligned}
\left\|T_{n} f\right\|_{v} \leq & 2 b d^{2} c_{2}\left(M_{\infty}\left(T_{n-1} T_{n} f, s_{n-1}\right) v\left(s_{n-1}\right)\right. \\
& \left.M_{\infty}\left(T_{n}^{2} f, s_{n}\right) v\left(s_{n}\right), M_{\infty}\left(T_{n+1} T_{n} f, s_{n+1}\right) v\left(s_{n+1}\right)\right) \\
\leq & 2 b d^{2} c_{2}\left(\sup _{k}\left\|T_{k}\right\|\right) \sup \left(M_{\infty}\left(T_{n} f, s_{n-1}\right) v\left(s_{n-1}\right),\right. \\
& \left.M_{\infty}\left(T_{n} f, s_{n}\right) v\left(s_{n}\right), M_{\infty}\left(T_{n} f, s_{n+1}\right) v\left(s_{n+1}\right)\right) \\
\leq & \left.4 b^{2} d^{4} c_{2} \sup _{k}\left\|T_{k}\right\|\right) M_{\infty}\left(T_{n} f, s_{n}\right) v\left(s_{n}\right)
\end{aligned}
$$

In the last inequality we used Lemma 3.1 of [6] again.

The boundedness of the Riesz projection is proven in Proposition 6.8 of $[6]$.

\section{Main result with examples}

Assume that a weight $v$ on $\Omega=\mathbb{D}$ or $\mathbb{C}$, satisfying $(B)$, is given; let the sequences $\left(m_{n}\right)_{n=1}^{\infty}$ and $\left(s_{n}\right)_{n=1}^{\infty}$ and also the multiplier sequences $\left(t_{n k}\right)_{k=1}^{\infty}$ be as above. 
Given a continuous function $f$ on $\Omega$, let $f_{k}(r)$ be the $k$ th Fourier coefficient of $\left.f\right|_{r \partial \mathbb{D}}$, i.e.

$$
f_{k}(r)=\frac{1}{2 \pi} \int_{0}^{2 \pi} f\left(r e^{i \varphi}\right) r^{-|k|} e^{-i k \varphi} d \varphi .
$$

Hence, for $z \in \mathbb{D}$ and $r=|z|$, we have

$$
f(z)=\sum_{k} f_{k}(r) e_{k}(z)
$$

at least in the $L^{2}$-sense on every $r \partial \mathbb{D}$. We define for all $n$ the operators

$$
W_{n} f=W_{n} \sum_{k} f_{k} e_{k}:=\sum_{k} t_{n k} f_{k}\left(s_{n}\right) e_{k}
$$

Notice that the sum on the right hand side has only finitely many terms. We define the projection

$$
P_{C} f:=\sum_{n=1}^{\infty} R W_{n} f=\sum_{k \geq 0} \sum_{n=1}^{\infty} t_{n k} f_{k}\left(s_{n}\right) e_{k},
$$

provided the series in (16) converges at least uniformly on compact subsets of $\mathbb{D}$. (It is then the Taylor series of an analytic function.) This is true, if we can show that $\left\|P_{C} f\right\|_{v}<\infty$, see Theorem 2 below.

To define a corresponding projection $P_{M}$ on the larger space of bounded functions we choose for every $n$ the interval $I_{n}:=\left[s_{n}, s_{n}+\epsilon_{n}\right]$ with $\left.I_{n} \subset\right] 0,1\left[\right.$, if $\Omega=\mathbb{D}$, and $\left.I_{n} \subset\right] 0, \infty\left[\right.$, if $\Omega=\mathbb{C}$. Here $\epsilon_{n}$ is fixed so small that $v\left(s_{n}\right) \geq v(r) \geq v\left(s_{n}\right) / 2$ for all $r \in I_{n}$ (and that $\left.s_{n}+\epsilon_{n}<s_{n+1}\right)$. We define

$$
Z_{n} f:=Z_{n} \sum_{k} f_{k} e_{k}:=\sum_{k} \frac{t_{n k}}{\epsilon_{n}}\left(\int_{I_{n}} f_{k}(s) d s\right) e_{k},
$$

and, as in (16),

$$
P_{M} f:=\sum_{n=1}^{\infty} R Z_{n} f=\sum_{k \geq 0} \sum_{n=1}^{\infty} \frac{t_{n k}}{\epsilon_{n}}\left(\int_{I_{n}} f_{k}(s) d s\right) e_{k},
$$

provided the series converges at least uniformly on compact subsets of $\mathbb{D}$.

Theorem 2. Let $v$ satisfy $(B)$. Then $P_{M}$ is a bounded projection $L_{v}^{\infty} \rightarrow H v$, and $P_{C}$ is a bounded projection from $L_{v}^{\infty} \cap C(\Omega)$ onto $H v$.

Here $C(\Omega)$ is the space of all continuous functions $f: \Omega \rightarrow \mathbb{C}$. 
Remark 2. Consider the case $\Omega=\mathbb{D}$. Then the condition $(B)$ is necessary and sufficient for Theorem 2 . Indeed, if $(B)$ does not hold, then $H v$ is isomorphic to $H^{\infty}$, by [6]. In this case $H v$ cannot be complemented in $L_{v}^{\infty}$ and hence, in particular, $P_{M}$ cannot be bounded.

Proof $1^{\circ}$. We start by showing that $P_{M}$ and $P_{C}$ are bounded. Concerning $P_{M}$, we want to prove first

$$
M_{\infty}\left(Z_{n} f, s_{n}\right) v\left(s_{n}\right) \leq C\left\|T_{n} f\right\|_{v}
$$

for $f \in L_{v}^{\infty}$. For $f(z)=\sum_{k} f_{k}(r) e_{k}(z)$ we have

$$
\begin{gathered}
M_{\infty}\left(Z_{n} f, s_{n}\right) v\left(s_{n}\right)=\operatorname{ess} \sup _{\varphi \in[0,2 \pi]}\left|\sum_{k \in \mathbb{Z}} \frac{t_{n k}}{\epsilon_{n}}\left(\int_{I_{n}} f_{k}(s) d s\right) s_{n}^{|k|} e^{i k \varphi}\right| v\left(s_{n}\right) \\
\leq \text { ess } \sup _{\varphi \in[0,2 \pi]}\left|\frac{1}{\epsilon_{n}} \int_{I_{n}} \sum_{k \in \mathbb{Z}} t_{n k} f_{k}(s) s_{n}^{|k|} e^{i k \varphi} d s\right| v\left(s_{n}\right) \\
\leq \operatorname{ess} \sup _{\varphi \in[0,2 \pi]} \sup _{s \in I_{n}}\left|\sum_{k \in \mathbb{Z}} t_{n k} f_{k}(s) s_{n}^{|k|} e^{i k \varphi}\right| v\left(s_{n}\right) \\
(20) \quad=\sup _{s \in I_{n}} \sup _{\varphi \in[0,2 \pi]}\left|\sum_{k \in \mathbb{Z}} t_{n k} f_{k}(s) s_{n}^{|k|} e^{i k \varphi}\right| v\left(s_{n}\right) .
\end{gathered}
$$

We obtain

$$
\sup _{\varphi \in[0,2 \pi]}\left|\sum_{k \in \mathbb{Z}} t_{n k} f_{k}(s) s_{n}^{|k|} e^{i k \varphi}\right| \leq \sup _{\varphi \in[0,2 \pi]}\left|\sum_{k \in \mathbb{Z}} t_{n k} f_{k}(s) s^{|k|} e^{i k \varphi}\right|
$$

for every $s \in I_{n}$. (To see this, we have $r_{k} \leq s$ for these $s$. We apply the maximum modulus principle to the harmonic function $h\left(r e^{i \varphi}\right)=$ $\sum_{k \in \mathbb{Z}} t_{n k} f_{k}(s) r^{|k|} e^{i k \varphi}$ to get $\left.\sup _{\varphi}\left|h\left(s_{k} e^{i \varphi}\right)\right| \leq \sup _{\varphi}\left|h\left(s e^{i \varphi}\right)\right|.\right)$

By the choice of the interval $I_{n}, v\left(s_{n}\right) \leq 2 v(s)$ for $s \in I_{n}$. Using these we obtain (19): we bound (20) by

$$
2 \sup _{s \in I_{n}} \sup _{\varphi \in[0,2 \pi]}\left|\sum_{k \in \mathbb{Z}} t_{n k} f_{k}(s) s^{|k|} e^{i k \varphi}\right| v(s) \leq 2\left\|T_{n} f\right\|_{v} .
$$

Let $f \in L_{v}^{\infty}$ be arbitrary. Notice that $Z_{n} f$ is always of the form $T_{n} g$ for some $g \in h v$ : we can write the right hand side of (17) as

$$
T_{n} \sum_{|k| \leq N_{n}} \frac{1}{\epsilon_{n}} \int_{I_{n}} f_{k}(s) d s e_{k}
$$


for an $N_{n} \in \mathbb{N}$ large enough. We thus can use (10) to obtain

$$
\begin{aligned}
\left\|P_{M} f\right\|_{v} & \leq\|R\| \cdot\left\|\sum_{n} Z_{n} f\right\|_{v} \\
\leq & d_{2}\|R\| \sup _{n} M_{\infty}\left(T_{n} Z_{n-1} f+T_{n} Z_{n} f+T_{n} Z_{n+1} f, s_{n}\right) v\left(s_{n}\right) \\
\leq & d_{2}\|R\|\left(\sup _{k}\left\|T_{k}\right\|\right) \sup _{n}\left(M_{\infty}\left(Z_{n-1} f, s_{n}\right) v\left(s_{n}\right)+\right. \\
& \left.M_{\infty}\left(Z_{n} f, s_{n}\right) v\left(s_{n}\right)+M_{\infty}\left(Z_{n+1} f, s_{n}\right) v\left(s_{n}\right)\right) .
\end{aligned}
$$

This is bounded by a $v$-dependent constant times $\sup _{n}\left\|Z_{n} f\right\|_{v}$. Since $Z_{n} f=T_{n} g$ for some $g \in h v$, the second inequality of Theorem 1, (9), applies and yields, together with (19), the bound

$$
C \sup _{n} M_{\infty}\left(Z_{n} f, s_{n}\right) v\left(s_{n}\right) \leq C^{\prime} \sup _{n}\left\|T_{n} f\right\|_{v} \leq C^{\prime}\left(\sup _{n}\left\|T_{n}\right\|\right)\|f\|_{v} .
$$

By Remark 1, (iii), the right hand side of (18) is the Taylor series of an analytic function and hence $P_{M} f$ is well-defined.

The proof for $P_{C}$ is easier, since the analogue of (19) is quite trivial:

$$
M_{\infty}\left(W_{n} f, s_{n}\right) v\left(s_{n}\right)=\sup _{|z|=s_{n}}\left|\sum_{k \in \mathbb{Z}} t_{n k} f_{k}(z) e_{k}(z)\right| v(z) \leq\left\|T_{n} f\right\|_{v} .
$$

The rest of the proof goes as that for $P_{M}$.

$2^{\circ}$. We show that $P_{M}$ and $P_{C}$ are projections. To this end fix $f \in L_{v}^{\infty}$ and fix $n$. Put $h=R Z_{n} f$. Then we have $h=\sum_{k=\left[m_{n-1}\right]}^{\left[m_{n+1}\right]} h_{k} e_{k}$ for certain constants $h_{k}$ and hence $Z_{n} h=T_{n} h$ for any $n$. We obtain

$$
\begin{aligned}
P_{M} h & =\sum_{k=1}^{\infty} R T_{k} h \\
& =T_{n-1} h+T_{n} h+T_{n+1} h \\
& =\left(V_{m_{n+2}, m_{n+1}}-V_{m_{n-1}, m_{n-2}}\right) h=h
\end{aligned}
$$

This proves that $P_{M}$ is a projection. The proof for $P_{C}$ is the same, replacing $Z_{n}$ by $W_{n}$.

Example 1. If $\Omega:=\mathbb{D}$ and $v(z)=\exp (-1 /(1-r))$, then

$$
r_{m}:=1-\frac{2}{1+\sqrt{4 m+1}} \cong 1-\frac{1}{\sqrt{m}}
$$


and the sequence $\left(m_{n}\right)_{n=1}^{\infty}$ is defined by $m_{n+1}:=m_{n}+\mathcal{O}\left(m_{n}^{3 / 4}\right)$ for all $n$. We refer to Example 2.3 of [6].

Example 2. If $\Omega:=\mathbb{C}$ and $v(z)=\exp \left(-e^{r}\right)$, then the number $r_{m}$ is the solution of the equation $x e^{x}=m$, i.e. $x+\log x=\log m$, hence, $r_{m}$ equals $\log m+$ very small corrections. The sequence $\left(m_{n}\right)_{n=1}^{\infty}$ is obtained from $m_{n+1}:=m_{n}+\mathcal{O}\left(\sqrt{m_{n}} \log m_{n}\right)$. For details, see [6], Example 2.1.

Example 3. If $\Omega:=\mathbb{C}$ and $v(z)=\exp \left(-(\log r)^{\rho}\right),|z| \geq 1, \rho \geq 2$ fixed, and $v(z)=1$ for $|z|<1$ then

$$
r_{m}:=\exp \left((n / \rho)^{1 /(\rho-1)}\right)
$$

and the sequence $\left(m_{n}\right)_{n=1}^{\infty}$ is defined by $m_{n+1}:=m_{n}+\beta m_{n}^{(\rho-2) /(\rho-1)}$ for a positive constant $\beta$. See [6], Example 2.2.

We finish our paper by the remark that in the case $\Omega=\mathbb{D}$ the condition $(B)$ can be described in a somewhat easier way. We have

Proposition 1. Let $\Omega=\mathbb{D}$. Then the following are equivalent:

(i) Condition (B)

(ii) $\forall b_{1}>1 \exists b_{2}>1 \forall m, n \geq 1$

$$
\left(\frac{r_{m}}{r_{n}}\right)^{m} \frac{v\left(r_{m}\right)}{v\left(r_{n}\right)} \leq b_{1} \Rightarrow\left(\frac{r_{n}}{r_{m}}\right)^{n} \frac{v\left(r_{n}\right)}{v\left(r_{m}\right)} \leq b_{2}
$$

Proof. We only have to show that condition $(B)$ implies (ii). To this end let $b_{1}>1$ and $m, n \geq 1$ be such that

$$
\left(\frac{r_{m}}{r_{n}}\right)^{m} \frac{v\left(r_{m}\right)}{v\left(r_{n}\right)} \leq b_{1}
$$

We show that there exists $b_{2}$, independent of $m$ and $n$, such that $\left(r_{n} / r_{m}\right)^{n-m} \leq b_{2}$. This implies (ii). Indeed, then we obtain

$$
\left(\frac{r_{n}}{r_{m}}\right)^{n} \frac{v\left(r_{n}\right)}{v\left(r_{m}\right)}=\left(\frac{r_{n}}{r_{m}}\right)^{n-m} \frac{r_{n}^{m} v\left(r_{n}\right)}{r_{m}^{m} v\left(r_{m}\right)} \leq\left(\frac{r_{n}}{r_{m}}\right)^{n-m} \leq b_{2} .
$$

Let $c=c\left(b_{1}\right)$ be the constant of condition $(B)$. Recall, that here we have $r_{k}<1$ for all $k$. We consider several cases.

If $m \geq c, n \geq c$ and $|m-n| \geq c$ then $(B)$ implies the existence of a constant $b_{3}$, independent of $m$ and $n$, with

$$
\left(\frac{r_{n}}{r_{m}}\right)^{n} \frac{v\left(r_{n}\right)}{v\left(r_{m}\right)} \leq b_{3}
$$


and hence $\left(r_{n} / r_{m}\right)^{n-m} \leq b_{1} b_{3}$.

If $|m-n|<c$ then $\left(r_{n} / r_{m}\right)^{n-m} \leq r_{1}^{-c}$ where the right-hand side is independent of $m$ and $n$.

Fix $N$ such that $r_{c+N}>r_{c}$. If $m \leq c+N$ and $n \leq c+N$ then similarly $\left(r_{n} / r_{m}\right)^{n-m} \leq r_{1}^{-2 c-2 N}$.

Now consider the case $n<c<c+N \leq m$. We have

$$
\begin{aligned}
\left(\frac{r_{c+N}}{r_{c}}\right)^{m-c-N} & \leq\left(\frac{r_{c+N}}{r_{n}}\right)^{m-c-N} \frac{r_{c+N}^{c+N} v\left(r_{c+N}\right)}{r_{n}^{c+N} v\left(r_{n}\right)} \\
& \leq\left(\frac{r_{c+N}}{r_{n}}\right)^{m-c-N}\left(\frac{r_{c+N}}{r_{n}}\right)^{c+N-m}\left(\frac{r_{m}}{r_{n}}\right)^{m} \frac{v\left(r_{m}\right)}{v\left(r_{n}\right)} \\
& \leq b_{1} .
\end{aligned}
$$

Since $\lim _{k \rightarrow \infty}\left(r_{c+N} / r_{c}\right)^{k-c-N}=\infty$ we find $k_{0}$, independent of $m$ and $n$, such that $\left(\frac{r_{c+N}}{r_{c}}\right)^{k-c-N}>b_{1}$ for all $k \geq k_{0}$. Hence $m \leq k_{0}$ and we obtain

$$
\left(\frac{r_{n}}{r_{m}}\right)^{n-m}=\left(\frac{r_{m}}{r_{n}}\right)^{m-n} \leq\left(\frac{1}{r_{1}}\right)^{k_{0}}
$$

Finally, assume $m<c<c+N \leq n$. Since $\lim _{l \rightarrow \infty} v\left(r_{l}\right)=0$ we find $l_{0}$ such that $r_{l}^{j} v\left(r_{l}\right) \leq v\left(r_{l}\right)<r_{c}^{c} v\left(r_{c}\right) / b_{1}$ for all $j>0$ and all $l \geq l_{0}$. Since

$$
\frac{r_{c}^{c} v\left(r_{c}\right)}{r_{n}^{m} v\left(r_{m}\right)} \leq r_{c}^{c-m} \frac{r_{m}^{m} v\left(r_{m}\right)}{r_{n}^{m} v\left(r_{n}\right)} \leq b_{1}
$$

we have $r_{c}^{c} v\left(r_{c}\right) / b_{1} \leq r_{n}^{m} v\left(r_{n}\right)$. Hence $n \leq l_{0}$ and we obtain $\left(r_{n} / r_{m}\right)^{n-m} \leq$ $r_{1}^{-l_{0}}$.

Now put $b_{2}=\max \left(b_{1} b_{3}, r_{1}^{-\max \left(2 c+2 N, l_{0}, k_{0}\right)}\right)$. Then we have in any case $\left(r_{n} / r_{m}\right)^{n-m} \leq b_{2}$.

\section{References}

[1] D. Békollé, Inégalités à poids pour le projecteur de Bergman dans la boule unité de $\mathbf{C}^{n}$, Studia Math., 71 (1981/82), 305-323.

[2] J. Bonet, M. Englis and J. Taskinen, Weighted $L^{\infty}$-estimates for Bergman projections, Studia Math., 171 (2005), 67-92.

[3] A. Harutyunyan and W. Lusky, Toeplitz operators on weighted spaces of holomorphic functions, submitted. 
[4] T. Lindenstrauss, Classical Banach Spaces I, Sequence Spaces, Ergebnisse der Mathematik und ihrer Grenzgebiete 92, Springer Verlag, 1977.

[5] D. Luecking, Representation and duality in weighted spaces of analytic functions, Indiana Univ. Math. J., 34 (2) (1985), 319-336. Erratum. Indiana Univ. Math. J. 35 (4) (1986), 927-928.

[6] W. Lusky, On the isomorphism classes of weighted spaces of harmonic and holomorphic functions, Studia Math., 175 (2006), 19-45.

[7] J. Taskinen, Regulated domains and Bergman type projections, Ann. Acad. Sci. Fenn., 28 (2003), 55-68.

[8] K. Zhu, Operator Theory in Function Spaces, Marcel Decker, New York (1995).

Fachbereich 17, Mathematik und Informatik

Universität Paderborn

D-33098 Paderborn

Germany

(E-mail : lusky@uni-paderborn.de)

Department of Mathematics and Statistics

University of Helsinki

P.O.Box 68, FIN-00014 Helsinki

Finland

(E-mail : jari.taskinen@helsinki.fi)

(Received : March 2007) 


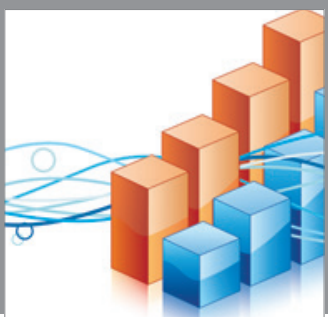

Advances in

Operations Research

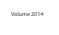

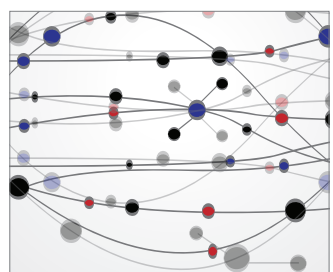

\section{The Scientific} World Journal
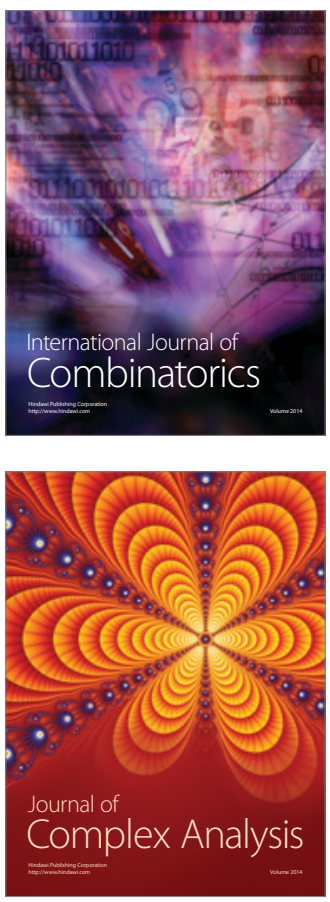

International Journal of

Mathematics and

Mathematical

Sciences
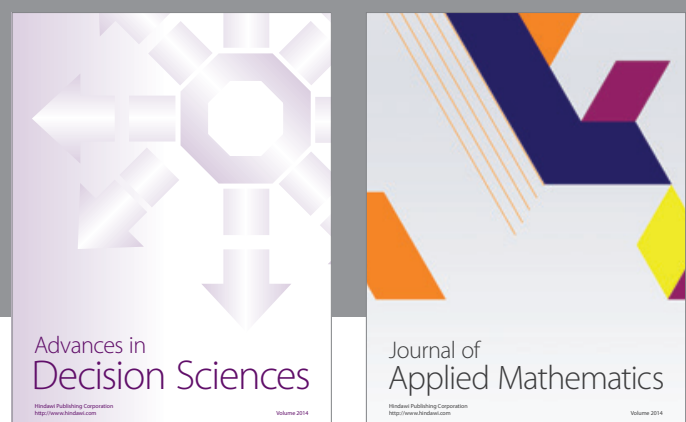

Journal of

Applied Mathematics
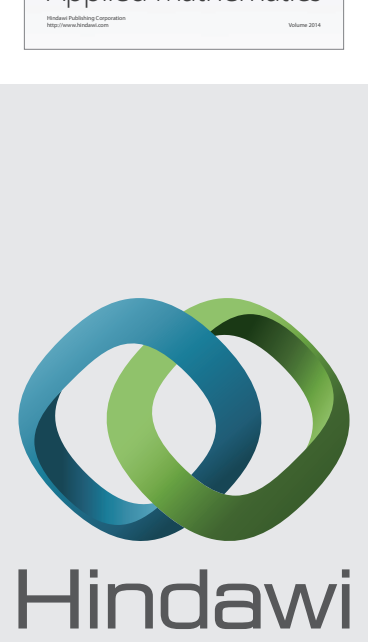

Submit your manuscripts at http://www.hindawi.com
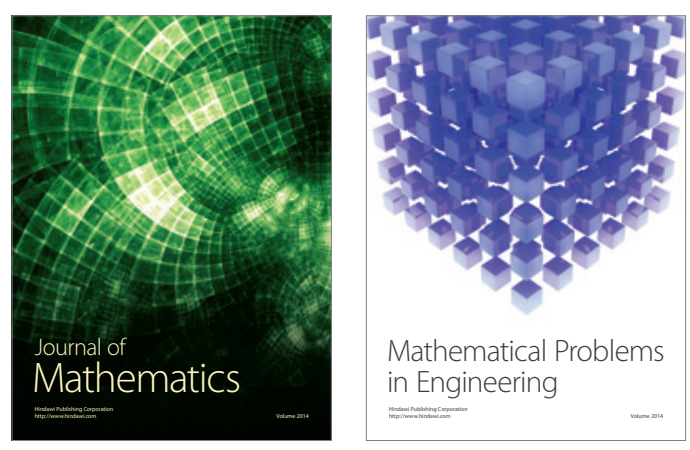

Mathematical Problems in Engineering
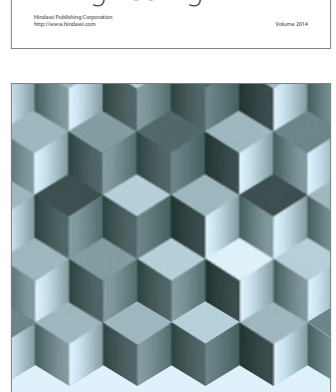

Journal of

Function Spaces
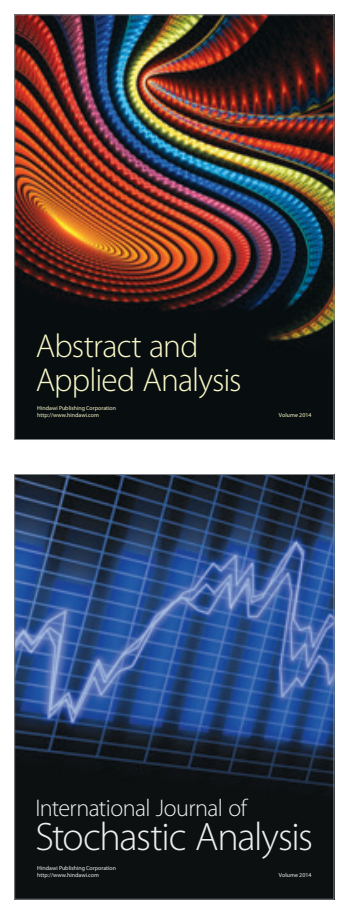

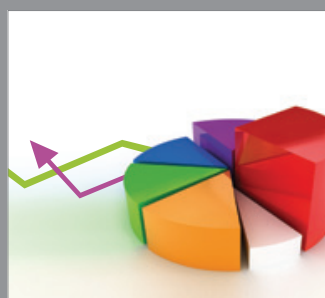

ournal of

Probability and Statistics

Promensencen
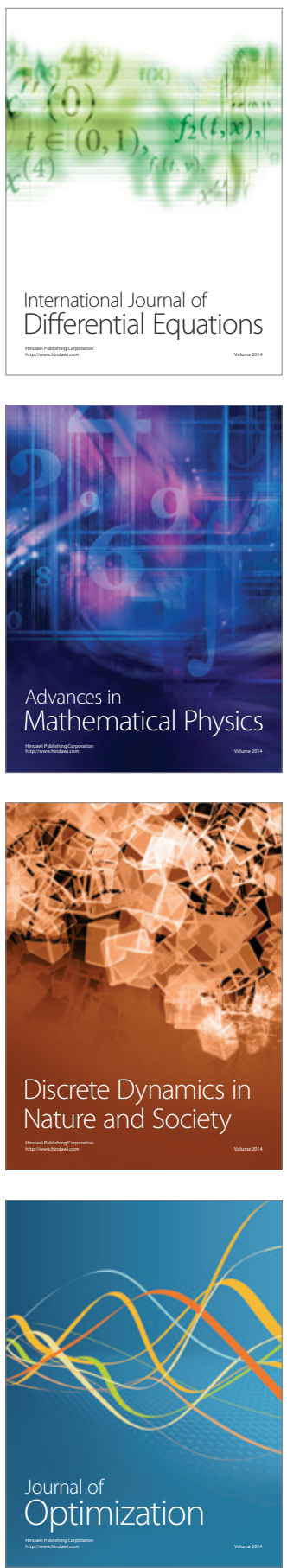